\title{
Research on VNIR Spectra of Petroleum Hydrocarbon in Contaminated Soil
}

\author{
Wenqi Yin ${ }^{1}$, Zhili Chen ${ }^{1}$, Hongtao Liu ${ }^{2}$, Qiang Liu ${ }^{2}$, and Yi Yang ${ }^{3}$ \\ ${ }^{1}$ Department of defense architectural planning and environmental engineering of Logistic \\ Engineering University of PLA, Chongqing, 401311; \\ ${ }^{2}$ Department of military oil application and management engineering of Logistic Engineering \\ University of PLA, Chongqing, 401311; \\ ${ }^{3}$ Department of military engineering management of Logistic Engineering University of PLA, \\ Chongqing, 401311
}

\begin{abstract}
Leakage of oil and petroleum products can cause serious soil pollution. Traditional monitoring method for soil petroleum hydrocarbon pollution finds it difficult to diagnose a large area of soil contaminated area, while visible and near infrared (VNIR) spectroscopy technology boasts advantages like fast and convenient. Therefore, it is the most promising means to rapid acquisition of soil information, and the trend of future research. The experiments are designed to simulate different degrees of pollution under the actual conditions, and the spectral characteristics and differences of the different types of petroleum hydrocarbon contaminated soil are measured and analyzed by the method of VNIR spectroscopy. The study shows: (1) When the content of petroleum hydrocarbon is the same, the spectral reflectance of engine oil contaminated soil is lower than that of diesel oil and gasoline contaminated soil; spectral response characteristics of soil containing diesel oil and engine oil are in the depth of $1206 \mathrm{~nm}$ and $1728 \mathrm{~nm}$, which spectral response characteristics of soil containing gasoline is in the depth of $1192 \mathrm{~nm}$ and $1707 \mathrm{~nm}$. And their absorption depths in the response characteristics are as engine oil, gasoline and diesel oil, from big to small. (2) With the increase of petroleum hydrocarbon pollution, the absorption depth of water absorption Valley at $1423 \mathrm{~nm}$ decreases first and then increases, which can be used as an auxiliary basis to identify soil petroleum hydrocarbon pollution.
\end{abstract}

Keywords: Soil; Oil hydrocarbon pollution; Spectral; Response characteristics

\section{土壤石油烃污染的可见-近红外光谱特性研究}

\author{
尹文琦 ${ }^{1}$ ，陈志莉 ${ }^{1}$ ，刘洪涛 ${ }^{2}$ ，刘强 ${ }^{2}$ ，杨毅 ${ }^{3}$ \\ (1. 后勤工程学院国防建筑规划与环境工程系, 重庆 401311; 2. 后勤工程学院军事油料应用与管理工程系,
} 重庆 401311;3.后勤工程学院军事工程管理系，重庆 401311)

摘要: 石油和石油产品的泄漏可造成严重的土壤污染, 传统的土壤石油烃污染监测方法难以满足大面积诊断土壤污染区 域的需求，而可见-近红外光谱技术具有快速、便捷等优势，是土壤信息快速获取最有潜力的手段，也是未来研究发展的趋 势。在实验室设计了模拟实际条件下不同程度污染的实验，采用可见-近红外光谱方法测量并分析了不同类别石油烃污染土 壤的光谱特性及其差异, 研究表明: (1)石油烃含量相同时, 机油污染土壤的光谱反射率低于柴油和汽油污染土壤的; 含有 柴油和机油的土壤光谱响应特征在 $1206 \mathrm{~nm}$ 和 $1728 \mathrm{~nm}$ 处, 而含有汽油的土壤光谱响应特征在 $1192 \mathrm{~nm}$ 和 $1707 \mathrm{~nm}$ 处, 而且 响应特征处的吸收深度, 机油>汽油>柴油。(2)随着石油烃污染程度的增大, $1423 \mathrm{~nm}$ 处水分吸收谷的吸收深度先减小后增大, 该变化可以作为辅助依据识别土壤石油烃污染。

关键词: 土壤; 石油烃污染; 光谱; 响应特征

中图分类号: 0433 文献标志码: A

引言

全球石油工业的发展和石油产品销售的增长，发生溢油泄漏事故的概率增大。土壤的石油烃类污染长 期以来是一个严重的环境问题，不仅严重破坏生态环境，还威胁人和动物的健康 [1]。石油和石油产品的 泄漏往往发生在石油的开采、提炼、储存和运输过程中，由于操作不慎、设备的腐蚀和老化导致石油类物 
质进入土壤, 破坏土壤的组成和结构, 改变土壤的理化性质, 进而影响土壤的生产功能, 并且土壤中嗜烃 微生物的增长, 会导致氧气的耗竭、有机酸和 C02 的积累, 使得植被根部的呼吸受阻, 引起植被枯萎死亡。 因此如何及时快速地探测和评估石油烃类的泄漏污染是减小危害和降低损失的关键。传统的土壤石油烃类 污染检测方法需要大量土样的野外采集与实验室的测定, 不仅成本较高、耗时费力, 而且缺乏可便携性, 不能达到快速、大范围的识别和监测土壤石油烃类污染的目的。高光谱遥感技术的逐步发展为快速高效地 获取大范围区域土壤污染信息开辟了新的途径, 其中可见-近红外光谱技术具有快速、便携和无损等特点, 因其光谱分辨率高、数据信息丰富, 可利用地物反射光谱特征定量反演地物的物理化学性质, 已被广泛应 用于直接或间接获取土壤的多种性质 [2-4]。因此, 可见-近红外光谱技术具备传统检测土壤石油烃污染技 术无法比拟的优势。

本文以重庆紫色土分别与柴油、机油和汽油的混合物为研究对象, 通过在实验室模拟实际条件下不同 程度的污染并进行光谱测量，得出了不同含量下 3 种石油烃污染土壤的光谱曲线，分析了光谱曲线规律和 特性以及土壤石油烃污染的光谱响应特征, 从而为土壤石油烃污染的光谱探测与评估提供有效的基础依据 和技术参考。

\section{1 实验方法}

\section{1 实验样品}

实验土样为重庆地区表层 0-20cm 紫色土，除去土壤中的石块、植物残茬等，经过自然风干、研磨后 过 $2 \mathrm{~mm}$ 篮。实验油品为 $92 \#$ 汽油、0\#柴油和机油。

\section{2 光谱数据的获取}

\section{2 .1 光谱数据的采集}

用量筒取 $20 \mathrm{~cm} 3$ 的土壤放入直径 $7 \mathrm{~cm}$, 深 $1.5 \mathrm{~cm}$ 的有盖玻璃血内, 用塑料勺抹平，测定无油时纯土壤 的光谱; 为了研究 3 种石油烃在不同污染程度下的光谱特性, 用注射器分别抽取 $0.2 、 0.4 、 1.0 、 1.6 、 2.0$ 、 4. $0 、 10.0 、 16.0$ 和 $20.0 \mathrm{ml}$ 的柴油, 分别对应污染程度为 $1 \% 、 2 \% 、 5 \% 、 8 \% 、 10 \% 、 20 \% 、 50 \% 、 80 \%$ 和 $100 \%$, 将其注入 9 份土壤中, 用塑料勺搅拌均匀并抹平, 测量土壤与不同含量柴油混合物的光谱; 按照此法, 分 别抽取相同含量的汽油和机油注入土壤中，混合均匀并抹平，测量其光谱。

光谱测量采用美国 ASD 公司的 FieldSpec 3Hi-Res 型野外地物光谱仪，其波长范围是 $350 \sim 2500 \mathrm{~nm}$, 采样间隔为 $1.4 \mathrm{~nm}(350 \sim 1000 \mathrm{~nm})$ 和 $2 \mathrm{~nm}(1000 \sim 2500 \mathrm{~nm})$ 。在 $1000 \mathrm{~nm}$ 、 $1800 \mathrm{~nm}$ 附近的接缝处以及边缘波段 $350 \sim 399 \mathrm{~nm} 、 2451 \sim 2500 \mathrm{~nm}$ 处噪声较大, 其他波段信噪比高, 约为 $1000 ： 1$ 。光谱的测量在暗室进行, 光 源距土壤样品表面 $50 \mathrm{~cm}$, 天顶角 $45^{\circ}$, 采用 $25^{\circ}$ 视场角光纤探头, 位于土壤样本表面垂直上方 $15 \mathrm{~cm}$ 处。 每次测量之前先进行白板校正, 每个土样采集 10 条光谱曲线, 算术平均后得到该土样的实际反射光谱数 据 [5]。

\section{2 .2 光谱数据的预处理}

每个土样的光谱曲线去除噪声较大的边缘波段 $350 \sim 399 \mathrm{~nm}$ 和 $2451 \sim 2500 \mathrm{~nm}$ 波段。采用 Savitzky-Golay (SG) 滤波法对土壤光谱进行平滑去噪, SG 光滑去噪由 Unscrambler X10.4 软件实现 [6, 7]。

\section{2 .3 光谱吸收深度}

一条光谱曲线中光谱吸收的两个肩部 $\mathrm{A} 1$ 和 $\mathrm{A} 2$ 与光谱吸收谷点 $\mathrm{A}$ 共同构成了该曲线的光谱吸收特征, 
如图 1 所示。非吸收基线是 $\mathrm{A} 1$ 与 $\mathrm{A} 2$ 的连线 [8]。吸收谷点 $\mathrm{A}$ 与上述非吸收基线的垂直距离称作光谱吸收 深度 $(\mathrm{H})[9]$ 。

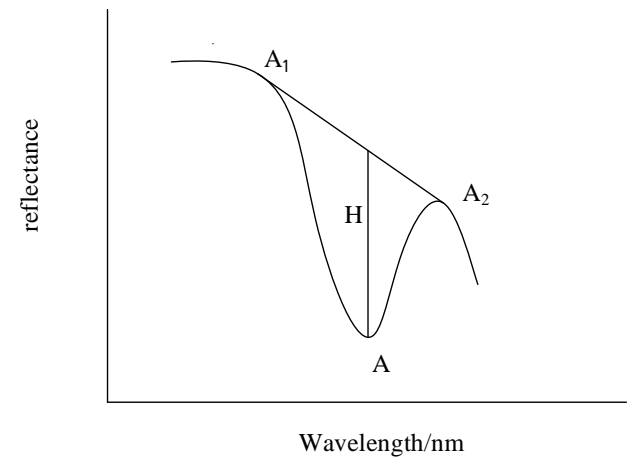

图 1 光谱吸收深度

\section{2 结果与讨论}

\section{1 不同污染浓度下土壤的光谱特征分析}

对注入不同含量的柴油、机油和汽油的土壤分别进行 10 次反射光谱测定取其平均值，得到 3 种含油 土壤光谱反射曲线, 如图 2、图 3 和图 4 所示。观察分析反射率曲线发现, 400 900nm 范围内, 随着石油 烃含量的增加, 光谱曲线由斜直形上升变为斜缓形上升, $900 \mathrm{~nm}$ 之后, 曲线基本保持平缓。对于注入柴油 和机油的土壤, 其光谱反射率在全波段范围内逐渐降低; 但注入汽油的土壤光谱规律却不同, 土壤反射率 并不是随着含量的增加而呈线性变化, 存在两处明显的差异, 分别是 $0.4 \mathrm{ml}$ 和 $2.0 \mathrm{~m} 1$ 。注入 $0.4 \mathrm{~m} 1$ 汽油的 土壤反射率高于 $0.2 \mathrm{ml}$ 时的反射率; 注入 $2.0 \mathrm{ml}$ 汽油的土壤反射率高于 $1.0 \mathrm{ml}$ 和 $1.6 \mathrm{ml}$ 时的反射率, 这可 能是因为注入 $1.0 \mathrm{~m} 1$ 和 $1.6 \mathrm{~m} 1$ 时, 附着在土壤颗粒表面的汽油对入射光起到吸收作用, 而 $2.0 \mathrm{~m} 1$ 时, 汽油 不仅覆盖在土壤颗粒上而且填充了土壤颗粒的间隙, 形成了一层很薄的油膜, 其镜面反射的作用大于吸收 作用, 所以反射率高于 $1.0 \mathrm{ml}$ 和 $1.6 \mathrm{ml}$ 时的反射率。土壤在注入 $2 \mathrm{ml}$ 柴油以后反射率下降幅度小, 曲线因 含量的增加而密集, 而加入 $2 \mathrm{ml}$ 机油和汽油后的土壤反射率下降幅度相比柴油较大。

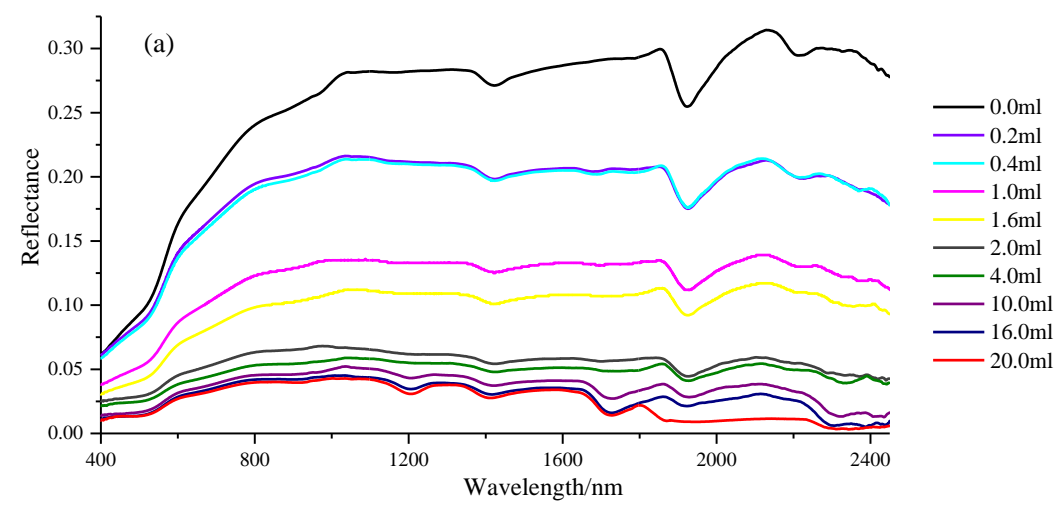




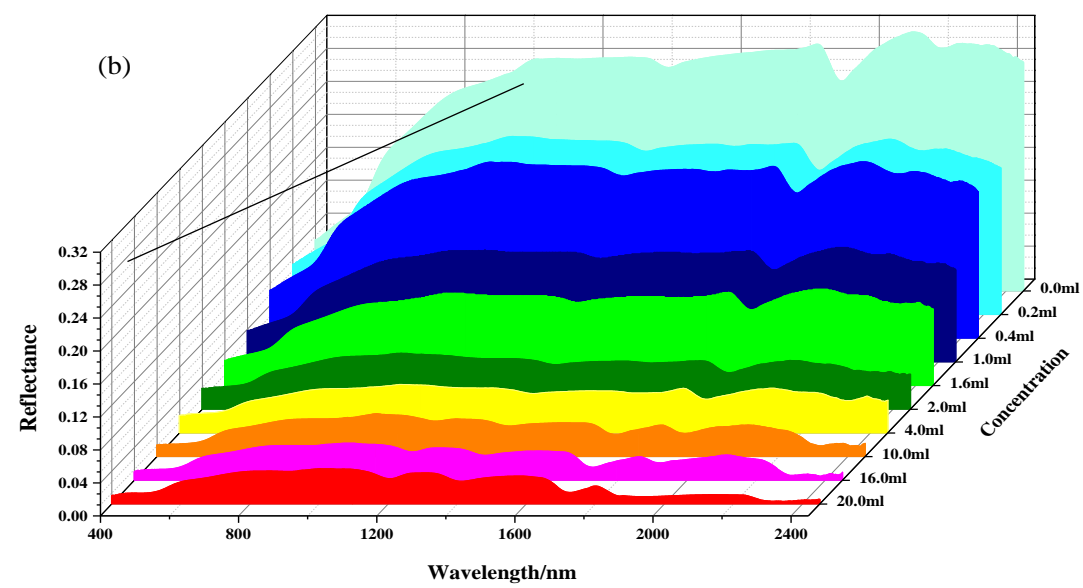

图 2 不同含量柴油污染土壤的光谱
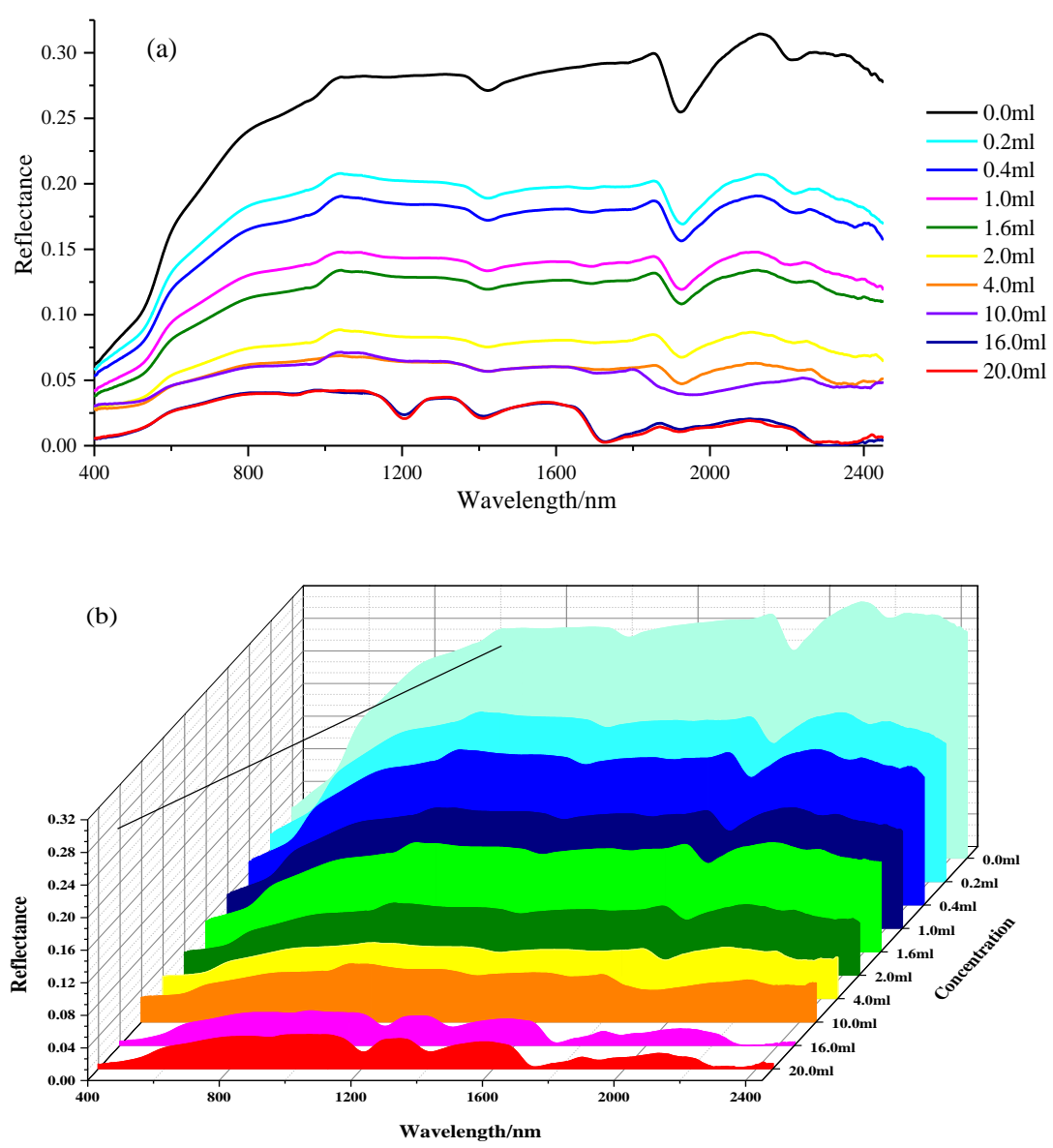

图 3 不同含量机油污染土壤的光谱 

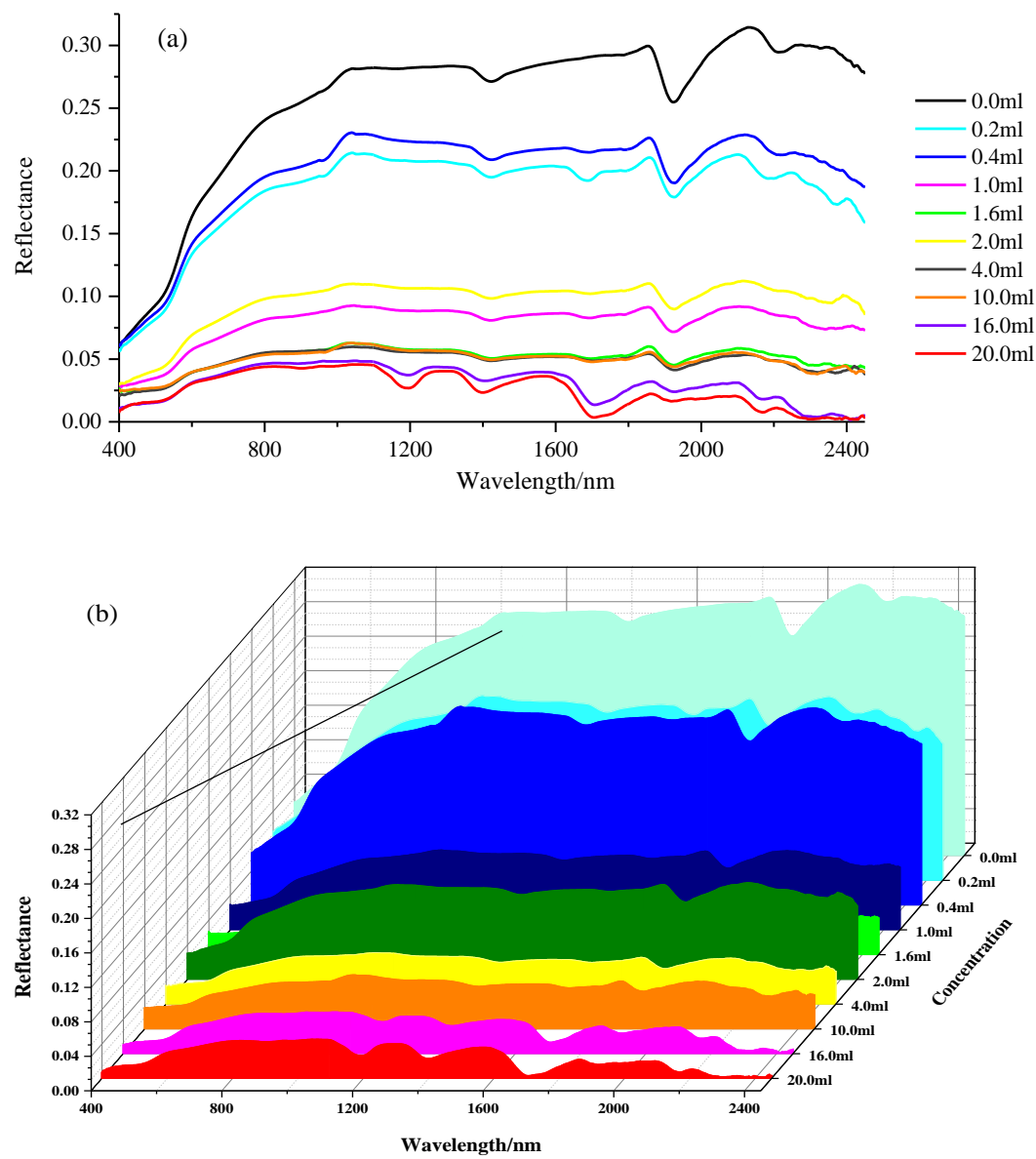

图 4 不同含量汽油污染土壤的光谱

针对柴油污染的土壤, 在注入 $0.2 \mathrm{ml}$ 和 $0.4 \mathrm{ml}$ 时土壤反射率相近, 光谱曲线发生重叠, 即当土壤被柴 油污染的程度为 $1 \%$ 和 $2 \%$ 时很难区分; 对于机油污染的土壤, 在注入 $16 \mathrm{~m} 1$ 和 $20 \mathrm{~m} 1$ 时反射率相近, 光谱曲 线发生重叠, 即当土壤被机油污染的程度为 $80 \%$ 和 $100 \%$ 时难以区分; 对于汽油污染的土壤, 在注入 $1.6 \mathrm{~m} 1$ 、 4. $0 \mathrm{ml}$ 和 $10.0 \mathrm{~m} 1$ 时反射率相近， 3 条光谱曲线基本重叠，即当土壤被汽油污染的程度为 $8 \%$ 、20\%和 50\%时 较难区分。

根据前人的研究成果，未被污染的土壤光谱曲线主要在 1400、1900、2200nm 附近存在明显的水分吸 收特征 ${ }^{[10-12]}$ 。观察 400-2450nm 波段范围的反射率可得, 整体的光谱曲线在 $1924 \mathrm{~nm}$ 处存在明显的吸收谷, 在 $1423 \mathrm{~nm}$ 和 $2213 \mathrm{~nm}$ 处有两处微小的吸收特征。随着石油烃污染程度的增加, 吸收特征波段并没有发生迁 移, 但是在逐渐减弱, 曲线趋于平坦, 1423 和 $2213 \mathrm{~nm}$ 处两个水分吸收特征都在注入量为 $2 \mathrm{ml}$ (污染程度为 $10 \%$ 时几乎消失， $1924 \mathrm{~nm}$ 处的水分吸收深度分别在注入柴油、机油和汽油为 $16 \mathrm{~m} 1 、 10 \mathrm{ml}$ 和 $20 \mathrm{~m} 1$ 时而变得 平缓。但 $1423 \mathrm{~nm}$ 波长处的水分吸收特征与 $1924 \mathrm{~nm}$ 和 $2213 \mathrm{~nm}$ 的有异同之处, 当石油烃污染达到 $80 \%$ 时又重 新显现, 并且吸收深度增大。

当柴油的注入量达到 $10 \mathrm{ml}$, 机油和汽油的注入量达到 $16 \mathrm{ml}$ 时, 光谱曲线产生了较大的差异, 在 $1200 \mathrm{~nm}$ 和 $1700 \mathrm{~nm}$ 附近出现了两个明显的吸收谷, 并且 $1700 \mathrm{~nm}$ 处的吸收深度较大, $1200 \mathrm{~nm}$ 和 $1700 \mathrm{~nm}$ 附近的响应 特征可以作为识别土壤中石油烃存在的依据。由此可得, 在土壤石油烃污染程度较低时, $1200 \mathrm{~nm}$ 和 $1700 \mathrm{~nm}$ 附近的响应特征表现不出来, 可能是被土壤中其他组分所掩盖, 只有当土壤石油烃污染到一定程度时, 可 
利用该识别依据诊断和监测土壤的石油烃污染。为了进一步确定该识别依据的具体波长位置, 需要提取相 同石油烃含量下土壤光谱数据进行分析。

\section{2 相同柴油、机油和汽油含量的土壤光谱特征分析}

分别提取 3 种石油烃含量为 $16 \mathrm{~m} 1$ 时的土壤光谱数据进行对比。观察图 5 中 3 条光谱, 在 $400 \sim 800 \mathrm{~nm}$ 波段范围, 3 条光谱曲线上升幅度相似，相同含量下，柴油与汽油的光谱曲线形状非常相似，而汽油的光 谱曲线比较特殊，与柴油的光谱曲线交互缠绕。含有机油的土壤光谱反射率整体低于含有柴油和汽油的光 谱反射率，而余璐等实验结果认为在相同的含量下，渗漏柴油的土壤反射率低于渗漏机油的土壤，本实验 的结论与其研究结果相反。

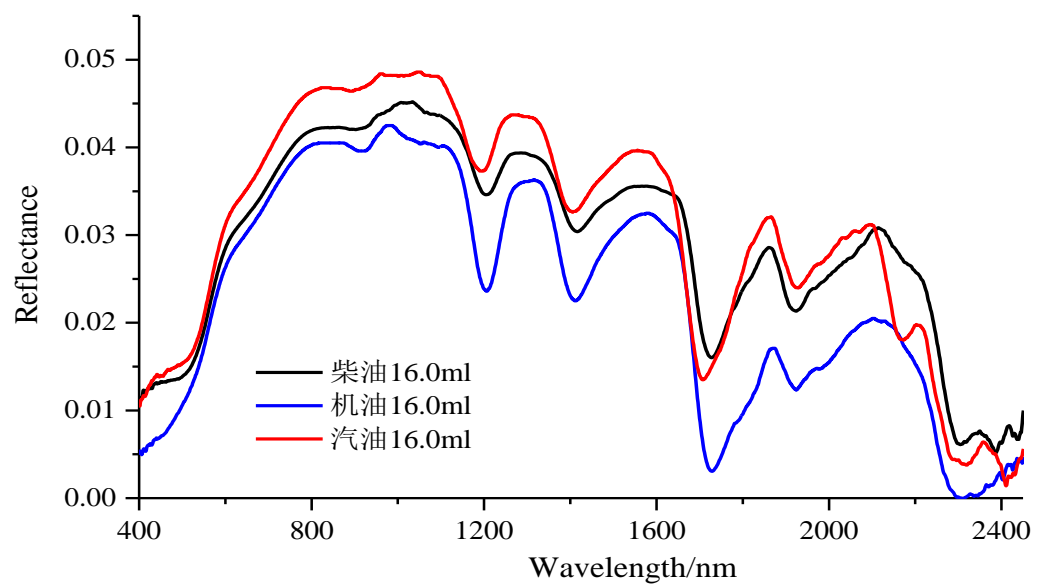

图 5 相同含量柴油、机油和汽油污染土壤的光谱

仔细观察 $1200 \mathrm{~nm}$ 和 $1700 \mathrm{~nm}$ 波长附近, 柴油和机油污染土壤的响应特征波段主要在 $1206 \mathrm{~nm}$ 和 $1728 \mathrm{~nm}$ 处，而汽油污染土壤的响应特征波段在 $1192 \mathrm{~nm}$ 和 $1707 \mathrm{~nm}$ 处。为了更好地区分柴油和机油，分别计算特征 波段处的吸收深度。表 1 为柴油、机油和汽油污染土壤的特征波段的吸收深度。分析不同石油烃下土壤光 谱吸收深度的异同可知，吸收深度：机油 >汽油>柴油，这与余璐等的实验结果一致。从图中还可以发现， $1423 \mathrm{~nm}$ 处水分吸收深度与 $1200 \mathrm{~nm}$ 附近石油烃吸收深度相近, 因此, 可以将 $1700 \mathrm{~nm}$ 附近的石油烃响应特征 作为识别土壤石油烃污染的第一依据, $1200 \mathrm{~nm}$ 附近的石油烃响应特征作为第二识别依据, $1423 \mathrm{~nm}$ 处水分 吸收特征的变化作为辅助识别依据。

表 1 柴油、机油和汽油污染土壤的特征波段的吸收深度

\begin{tabular}{lcccccc}
\hline & \multicolumn{2}{c}{ 柴 油 } & \multicolumn{2}{c}{ 机 油 } & \multicolumn{3}{c}{ 汽 油 } \\
\hline 特征波段 $/ \mathrm{nm}$ & 1728 & 1206 & 1728 & 1206 & 1707 & 1192 \\
吸收深度 $(\mathrm{H})$ & 0.0161 & 0.0056 & 0.0220 & 0.0127 & 0.0211 & 0.0078 \\
\hline
\end{tabular}

\section{3 结论}

本文测定和分析了重庆紫色土与不同体积的柴油、机油和汽油混合物的光谱反射率曲线，得出不同石 油烃污染的土壤光谱响应特征以及石油烃含量对土壤光谱特征的影响规律。研究结果表明, 位于 $1206 \mathrm{~nm}$ 
和 $1728 \mathrm{~nm}$ 处的吸收谷可以作为柴油和机油污染土壤的光谱响应特征, 而汽油污染土壤的光谱响应特征位 于 $1192 \mathrm{~nm}$ 和 $1707 \mathrm{~nm}$ 处，通过计算和对比响应特征的吸收深度，可以便于区分土壤中的石油烃种类。但是 当土壤石油烃含量低于一定量时, 土壤中石油烃的光谱响应特征比较微弱, 只有达到一定的污染程度, 才 可以通过光谱特征来识别土壤石油烃污染, 但具体的临界污染程度还需要开展进一步的研究。此外, $1423 \mathrm{~nm}$ 处水分吸收特征的变化可以作为次依据来辅助识别。相同石油烃含量下，含有机油的土壤光谱反射率低于 含有柴油的光谱反射率, 这与前人的研究成果相异, 还需后续的研究来验证。在不同污染程度下土壤光谱 的研究中, 含有柴油、机油和汽油的光谱都存在曲线重叠, 导致难以区分不同的污染程度, 可能成为将来 通过光谱探测土壤石油烃污染的难题。

\section{4 致谢}

感谢国家自然科学基金项目 (21377166) 对本研究的支持。

\section{参考文献：}

[1] Grant C. Soils and human health. [J]. Journal of Environmental Quality, 2013, 42 (6) :1909-1910.

[2] Brown D J, Shepherd K D, Walsh M G, et al. Global soil characterization with VNIR diffuse reflectance spectroscopy [J]. Geoderma, 2006, $132(3-4): 273-290$.

[3] Rossel R A V, Walvoort D J J, Mcbratney A B, et al. Visible, near infrared, mid infrared or combined diffuse reflectance spectroscopy for simultaneous assessment of various soil properties[J]. Geoderma, 2006, $131(1-2): 59-75$.

[4] Vasques G M, Grunwald S, Sickman J 0. Modeling of soil organic carbon fractions using visible-near-infrared spectroscopy. [J]. Soil Science Society of America Journal, 2009, 73(1):176-184.

[5] 纪文君, 李爴, 李成学等. 基于全谱数据挖掘技术的土壤有机质高光谱预测建模研究 $[\mathrm{J}]$. 光谱学与光谱分析, 2012 (09) : 2393-2398.

[6] 郑小坡, 孙越君, 秦其明等. 基于可见光-短波红外波谱反射率的裸土土壤含水量反演建模 $[\mathrm{J}]$. 光谱学与光谱分 析, 2015, 35(8) : 2113-2118

[7] 乔星星, 冯美臣, 杨武德等. SG 平滑处理对冬小麦地上干生物量光谱监测的影响 $[\mathrm{J}]$. 山西农业科学, 2016 (10) : 1450-1454.

[8］童庆禧，张兵，郑兰芬．高光谱遥感:原理、技术与应用 [M]．高等教育出版社，2006.

[9] 余璐, 刘学斌, 刘贵忠等. 土壤漏油含量光谱预测实验研究与分析 [J]. 光谱学与光谱分析, 2016, 36 (4) :1116-1120.

[10] 纪文君，史舟，周清等. 几种不同类型土壤的 VIS-NIR 光谱特性及有机质响应波段 $[J]$. 红外与毫米波学报, 2012, 31(3) :277-282.

[11] 吴代晖, 范闻捷, 崔要奎等. 高光谱遥感监测土壤含水量研究进展 $[\mathrm{J}]$. 光谱学与光谱分析, 2010 (11) : 3067-3071.

[12］刘影，姚艳敏．土壤含水量高光谱遥感定量反演研究进展 [J]。中国农学通报，2016(07): 127-134.

\section{References}

[1] Grant C. Soils and human health. [J]. Journal of Environmental Quality, 2013, 42(6): 1909-1910.

[2] Brown D J, Shepherd K D, Walsh M G, et al. Global soil characterization with VNIR diffuse reflectance spectroscopy [J]. Geoderma, 2006, 132(3-4):273-290. 
[3] Rossel R A V, Walvoort D J J, Mcbratney A B, et al. Visible, near infrared, mid infrared or combined diffuse reflectance spectroscopy for simultaneous assessment of various soil properties[J]. Geoderma, 2006, 131(1-2):59-75.

[4] Vasques G M, Grunwald S, Sickman J O. Modeling of soil organic carbon fractions using visible-near-infrared spectroscopy.[J]. Soil Science Society of America Journal, 2009, 73(1):176-184.

[5] Ji Wenjun, Li Xi, Li Chengxue, and so on. Study on Hyperspectral Prediction Modeling for Soil Organic Matter Based on Mining Technology of Full Spectrum Data [J].Spectroscopy and Spectral Analysis,2012(09):2393-2398.

[6] Zheng Xiaopo, Sun Yue june, Qin Qiming, and so on. Inversion Modeling for Bare Soil Moisture Based on Reflectance of Visible Light-Wave Infrared Spectral [J].Spectroscopy and Spectral Analysis,2015,35(8):2113-2118.

[7] Qiao Xingxing, Feng Meichen, Yang Wude and so on. Effect of SG Smoothing Treatment to Spectral Monitoring of Dry Biomass Quantity in Winter Wheat Earth [J].Journal of Shanxi Agricultural Sciences,2016(10):1450-1454.

[8] Tong Qingxi, Zhang Bing, Zheng Lanfen. Hyperspectral Remote Sensing: Principles, Techniques and Applications [M].Higher Education Press, 2006.

[9] Yu Lu, Liu Xuebin, Liu Guizhong, and so on. Experimental Study and Analysis on Spectrum Prediction of Oil Spill Contents in Soil [J].Spectroscopy and Spectral Analysis, 2016, 36(4):1116-1120.

[10]Ji Wenjun, Shi Zhou, Zhou Qing, and so on. VIS-NIR Spectra Properties of Several Different Types of Soil and Organic Matter Response Band [J].Journal of Infrared and Millimeter Waves, 2012,31(3):277-282.

[11]Wu Daihui, Fan Wenjie, Cui Yaokui and so on. Research Progress on Hyperspectral Remote Sensing Monitoring of Soil Moisture [J].Spectroscopy and Spectral Analysis, 2010(11):3067-3071.

[12]Liu Ying, Yao Yanmin. Research Progress on Quantitative Retrieval of Hyperspectral Remote Sensing for SOIL Moisture [J].Chinese Agricultural Science Bulletin, 2016(07):127-134. 